\title{
Invasive Sino-Orbital Aspergillosis in an Immunocompetent Patient
}

\author{
Okolo $\mathrm{OM}^{1}$, Bot $\mathrm{GM}^{2}$, Onyedibe $\mathrm{IK}^{1}$, Shilong $\mathrm{DJ}^{2}$, Alfin $\mathrm{DJ}^{2}$, Adoga $\mathrm{AS}^{3}$, Dahal AS${ }^{1}$, Ogbe $\mathrm{ME}^{4}$, Egah $\mathrm{ZD}^{1}$, Binitie $\mathrm{OP}^{2}$.
}

${ }^{1}$ Department of Medical Microbiology, ${ }^{2}$ Division of Neurosurgery, Department of Surgery, ${ }^{3}$ Department of Ear Nose and Throat (ENT), and ${ }^{4}$ Department of Anaesthesia, College of Health Science, University of Jos, Nigeria.

*Corresponding Author: Okolo Mark Ojogba. Department of Medical Microbiology, University of Jos, Plateau State, Nigeria. Email: okolomark@gmail.com

\begin{abstract}
Invasive aspergillosis that involves intra-orbital and intracranial extension occurs in immunodeficient and immunocompetent patients resulting in significant morbidity and mortality. We report the case of a 38year old farmer who had recurrent fronto-ethmoidal mucocoele and proptosis of the left eye. She presented with a history of hypertension and no other significant findings on examination. Computer tomography scan of the brain showed a left fronto-orbital uniform contrast-enhancing extra-axial lesion with thickened peripheral capsule and an associated left fronto-orbital skull defect. Cerebrospinal fluid analysis did not show any sign of infection. Intra-operative biopsy sample showed cheesy material which on culture grew Aspergillus species that was identified further using molecular methods. Antifungal agents were used to treat the patient. The present case strongly suggests that it is possible to control intracranial aspergillosis with a combination of surgery and antifungal chemotherapy.
\end{abstract}

\section{Keywords: Invasive aspergillosis, Aspergillus,Immunocompetent, Fungal infection}

\section{INTRODUCTION}

spergillosis of the paranasal sinuses and orbit
usually is self-limiting with a good prognosis. In
contrast, invasive diseases may result in significant
morbidity and mortality from intra-orbital and
intracranial extension. Invasive disease occurs in
debilitated patients with decreased immunity such as
diabetes mellitus, Human Immunodeficiency Virus,
cytotoxic drugs users, etc. ${ }^{1.3}$ Intracranial extension often
results in mortality despite therapy. Nonetheless,
invasive sino-orbital aspergillosis may occur in
immunocompetent patients resulting in significant
morbidity and mortality. The standard treatment for
invasive sino-orbital diseases has been radical surgical intervention, including orbital exenteration, sinus excision, and aggressive intracranial debridement. However more recently, poor prognosis have been reported with a combination of limited surgical debridement and anti-fungal therapy particularly in immunocompetent patients due to late presentation to health care facility. We present a case of invasive sinoorbital aspergillosis with extension into the dura complicated by cheesy collection in an immunocompetent patient. The patient was successfully managed with surgical debridement and anti-fungal chemotherapy.

\section{Case report: history and examination}

A 38 year old female who had gross total resection of 
fronto-orbital lesion, with intracranial extension for about 3 years prior to presentation. However, she started having serous discharge from the operation site about one year after the surgery with associated proptosis of the left eye. There was no discharge from the nose, ear or contralateral eye. Her medical history included only hypertension. The patient had marked proptosis, limitation of extra ocular motility, ptosis, and a large discharging (left) supra orbital sinus. The patient is immunocompetent as revealed by nonreactive retroviral screening test. The other findings from the physical examination were not significant. The patient was admitted and reviewed by both Neurosurgery and the Otorynolaryngology team, and a computer tomography scan of the brain was performed prior to surgery.

\section{Hospital care}

The imaging study of the patient's brain showed a left frontoorbital uniformly contrast-enhancing extra-axial lesion with thickened peripheral capsule. (fig1a,1b,1c) There was an associated left fronto-orbital skull defect and thickening of the left maxillary sinus mucosa and calcifications.

A thorough medical examination and review of patient, including Human Immunodeficiency Virus screening, did not reveal evidence of immunodeficiency disorder or systemic fungal infection. Given the immunocompetence of the patient, chronic nature of the disease, clinical and radiologic findings, a diagnosis of Recurrent fronto-ethmoidal mucocoele with fistula was made and the patient was prepared for external frontoethmoidectomy. Intraoperatively, a left fronto-cutaneous fistula was seen, cheesy material with frontal sinus with extension to the dura as well as reactive dura was observed. The cheesy material was evacuated and sent to the laboratory, irrigation was carried out and haemostasis secured with electrocautery. A frontonasal stent was placed for two weeks to allow for epithelization and enhanced drainage from the frontal sinus to the nasal cavity to allow for healing of the fronto-cutaneous fistula.

\section{Microbiology and molecular examination}

Cultures of the cheesy material sample obtained were consistent with Aspergillus species (figure 2). Internal transcribed spacer (ITS) region gene sequencing was performed to reliably identify the organism. The universal primers for fungi $\mathrm{F}$ (5'-TCCGTAGGTGAACCTGCGG-3') and R(5'-TCCTCCGCTTATTGATATGC-3') was used and generating a sequence which on BLAST analysis matched Aspergillus aculeatus (GenBank accession number MN187974.1) was identified. Due to unavailability of antifungal drugs, patient was commenced empirically on intravenous fluconazole for two weeks and discharged home on oral voriconazole $(300 \mathrm{mg})$ twice daily. The patient is eight months post-surgery doing very great.

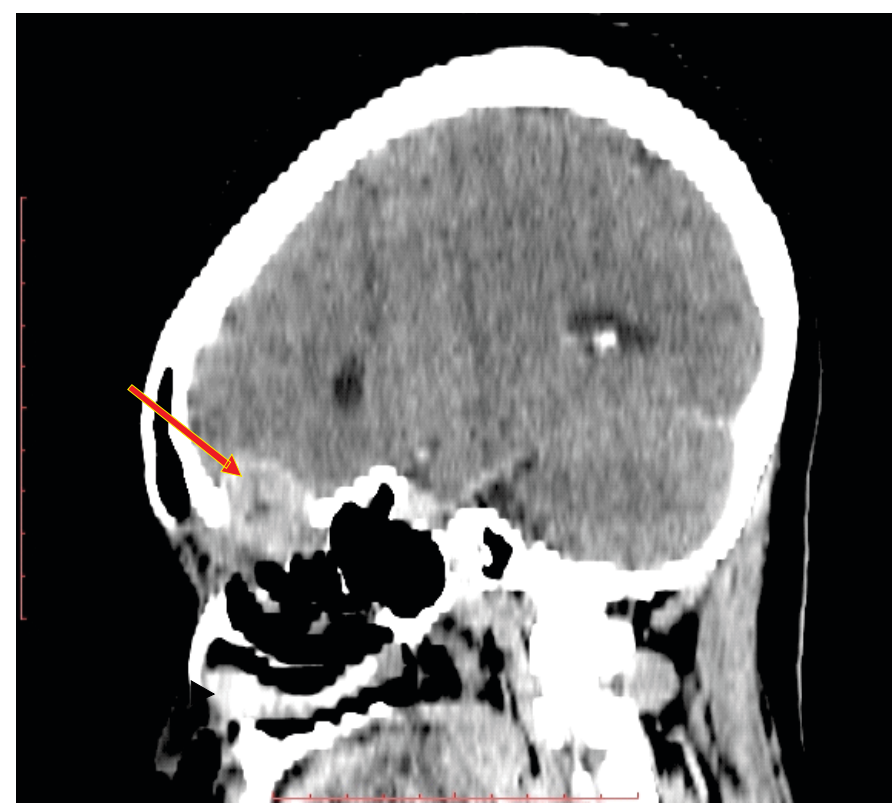

Fig 1a: Brain computer tomography scan showing lesion (arrow) in the fronto-nasal sinus.

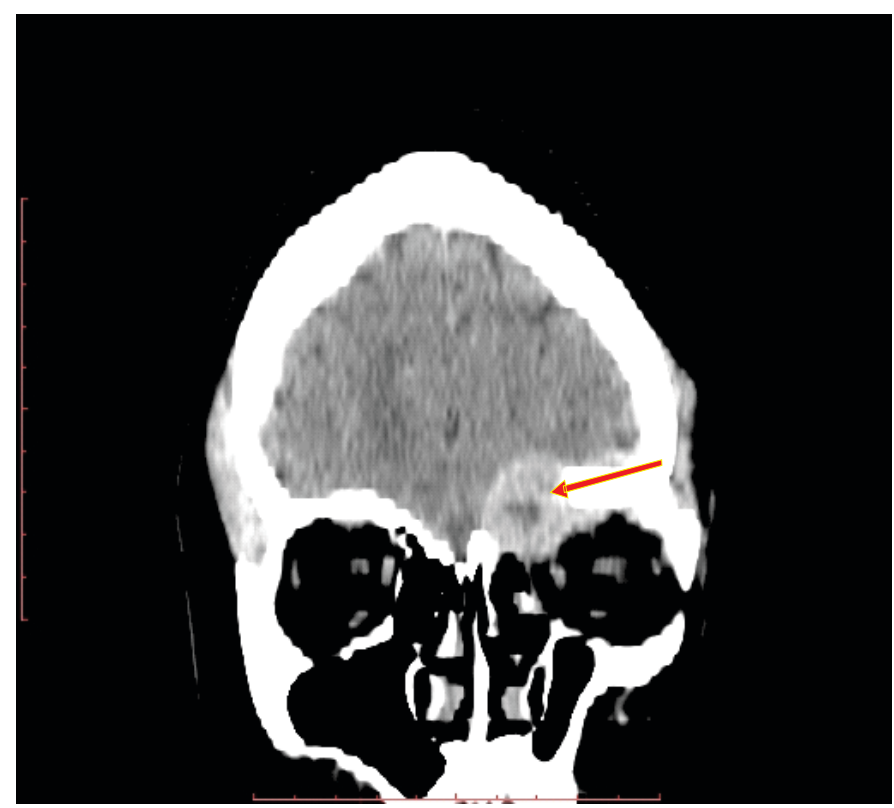

Fig 1b: Computer tomography scan of the brain showing(arrow) a left fronto-orbital uniform contrast-enhancing extra-axial lesion. 


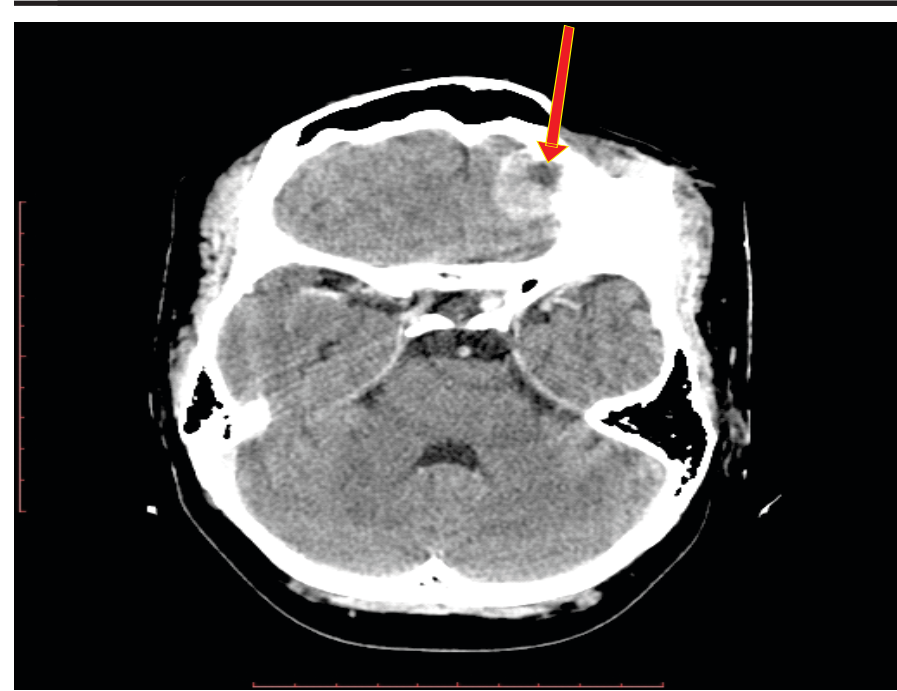

Fig1c: Computer tomography scan of the brain showing(arrow) a left fronto-orbital extra-axial lesion with thickened peripheral capsule and an associated left fronto-orbital skull defect

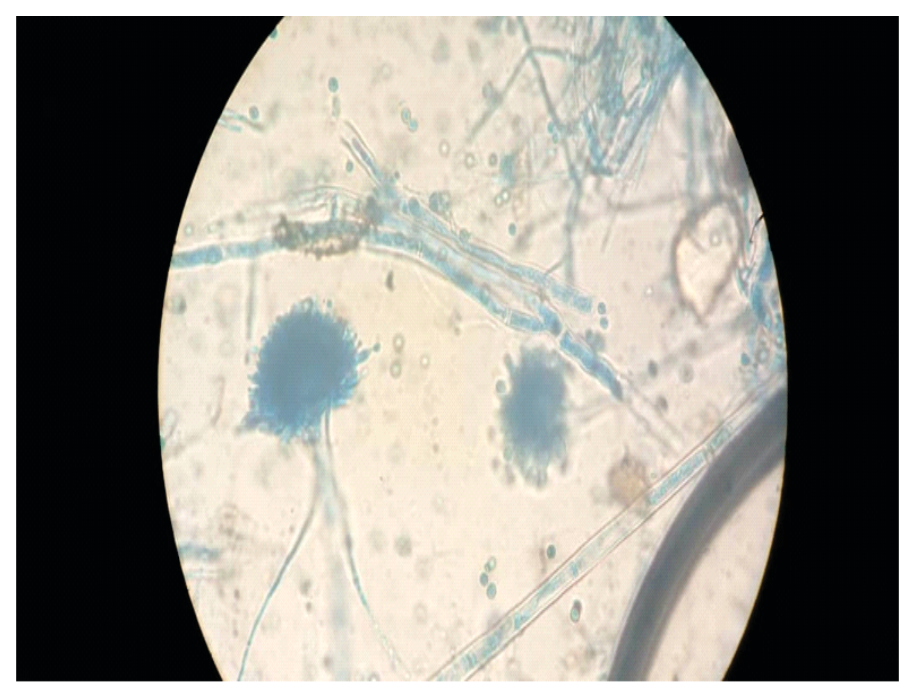

Fig2:Micrograph of Aspergillus species isolated from patient sample

\section{DISCUSSION}

Invasive sino-orbital aspergillosis is seen usually in immunodeficient patients and result in morbidity and mortality; it is rare in immunocompetent patients. Making a diagnosis of invasive sino-orbital aspergillosis in patients can be challenging particularly in low and middle income countries. Our patient had a chronic progression of lesion similarto findings by Sivak- Callcot and colleagues who reported a delay in diagnosis of up to 10 months because of nonspecific complaints of retro- orbital pain prior to ophthalmic features.
Several reports from previous studies showed that patients initially present with persistent unilateral, severe, frontal headache, or retro- orbital pain as their main presenting complaints. ${ }^{456}$ Some of the patients were diagnosed with temporal arteritis or orbital pseudo tumour and were treated with high dose systemic corticosteroids. ${ }^{789,10}$ Therefore, even in healthy patients, a complaint of persistent and/or escalating retro-orbital pain with or without discharging orbital sinus and/or ophthalmic features should prompt physicians to request orbital and cranial imaging studies before making a diagnosis and instituting treatment. Because the orbital findings are often non- specific, clinicians should have a high index of suspicion. Computed tomography scan findings of paranasal sinus and orbital mass and/or bone destruction (with or without optic nerve involvement) should be investigated by a biopsy to rule out fungal infection.

The pathophysiology of invasive aspergillosis in immunocompetent patients is not clear. It is believed that predisposing factors for both invasive and noninvasive types of infections include mechanical obstruction of nose and paranasal sinuses, such as septal abnormalities, orbital infections, and nasal polyps. The risk factor in our patient is orbital infection. Intracranial spread of fungus via bone erosion and migration along the blood vessels has been documented in previous studies. ${ }^{118}$ It is possible that the discharging orbital sinus was responsible for the infection and spread. Perhaps, early treatment with antifungal agent based on clinical and radiologic finding prior to surgery may reduce morbidity and mortality.

Management of invasive sino-orbital Aspergillosis in immunocompetent patients remains controversial. Management modality ranges from medical management alone to radical surgery with adjuvant chemotherapy.,12, Combination of antifungal agents has been used successfully to control infection in some studies..$^{14,15}$ Voriconazole is the drug of choice for invasive aspergillosis because patients demonstrate better tolerance for it and because of its lower toxicity compared to amphotericin B. Our patient had radical surgery with debridement followed with available parenteral fluconazole and then oral voriconazole to successfully control the disease. Due to the high rates of morbidity and mortality following radical debridement of invasive fungal infection, we believe that a combination of both surgery and medical chemotherapy may be reasonable in immunocompetent patients. 


\section{CONCLUSION}

Invasive sino-orbital Aspergillosis in immunocompetent patients is rare. We described a novel route of intracranial invasion in an immunocompetent patient with left eye proptosis and discharging orbital sinus. Prompt diagnosis of the infection is key to successful management of patients. Nonspecific complaints or retro-orbital pain with or without discharging orbital sinus should prompt a high index of suspicion. Radiologic findings with associated adjacent paranasal sinus involvement should lead physicians to consider urgent tissue biopsy to rule out aspergillosis. Our case shows that it is possible to control intracranial aspergillosis with a combination of surgery and antifungal chemotherapy in immunocompetent patients.

\section{Acknowledgement}

This research was supported by the Fogarty International Centre (FIC); Office of the Director (OD/NIH); National Institutes of Neurological Disorders and Stroke (NINDS/NIH); and the National Institute of Nursing Research (NINR/NIH) of the National Institutes of Health under award number D43TW010130. The content is solely the responsibility of the authors and does not necessarily represent the views of the National Institutes of Health

\section{Conflict of Interest}

None declared.

\section{REFERENCES}

1. Breen DJ, Clifton AG, Wilkins P, Uttley D, Westmore G. Invasive aspergilloma of the skull base. Neuroradiology. 1993;35(3):216-217.

2. Nenoff P, Kellermann S, Horn LC, Keiner S, Bootz F, Schneider S, et al. Case report. Mycotic arteritis due to Aspergillus fumigatus in a diabetic with retrobulbar aspergillosis and mycotic meningitis. Mycoses. 2001;44: 407-414.

3. Yoon JS, Park HK, Cho NH, Lee SY. Outcomes of three patients with intracranially invasive sino-orbital aspergillosis. Ophthal Plast Reconstr Surg. 2007;23(5):400-406.

4. Agarwal S, Kanga A, Sharma V, Sharma DR, Sharma ML. Invasive aspergillosis involving multiple paranasal sinuses--a case report. Indian J Med Microbiol. 2005 ;23(3):195-197.

5. Mylona S, Tzavara V, Ntai S, Pomoni M, Thanos L. Chronic invasive sinus aspergillosis in an immunocompetent patient: a case report

Dentomaxillofac Radiol. 2007;36(2):102-104.

6. Safdar A, Dommers MPJ, Talwani R, Thompson CR. Intracranial perineural extension of invasive mycosis: a novel mechanism of disease propagation by Aspergillus fumigatus. Clin Infect Dis. 2002;35(5):50-53.

7. Austin P, Dekker A, Kennerdell JS. Orbital aspergillosis. Report of a case diagnosed by fine needle aspiration biopsy. Acta Cytol. 1983;27(2):166-9.

8. Mcgill TJ, Simpson G, Healy GB. Fulminant aspergillosis of the nose and paranasal sinuses: A new clinical entity. Laryngoscope [Internet]. 1980;90(5):748-54. Available from: https://doi.org/10.1288/00005537-198005000-00003. Date accessed: 5 August 2019

9. Slavin ML. Primary Aspergillosis of the Orbital Apex. JAMA Ophthalmol [Internet]. 1991 Nov 1;109(11):1502-3. Available from: https://doi.org/10.1001/archopht.1991.0108011003602 4.Date accessed: 5 August 2019

10. Rafuse PE, Nicolle DA, Hutnik CML, Pringle CE. Left atrial myxoma causing ophthalmic artery occlusion. Eye [Internet]. 1997;11(1):25-9. Available from: https://doi.org/10.1038/eye.1997.5. Date accessed: 5 August 2019

11. Centeno RS, Bentson JR, Mancuso AA. CT scanning in rhinocerebral mucormycosis and aspergillosis. Radiology [Internet]. 1981 Aug 1;140(2):383-9. Available from: https://doi.org/10.1148/radiology.140.2.7255714.Date accessed: 6 August 2019

12. Panda NK, Saravanan K, Chakrabarti A. Combination antifungal therapy for invasive aspergillosis: can it replace high-risk surgery at the skull base? Am J Otolaryngol [Internet]. 2008;29(1):24-30. Available from:

http://www.sciencedirect.com/science/article/pii/S0196 070906003127.Date accessed: 6 August 2019

13. Parsonage MJ, Stafford ND, Lillie P, Moss PJ, Barlow $\mathrm{G}$, Thaker H. Oral voriconazole for invasive fungal skull base infection. J Laryngol Otol [Internet]. 2009/12/23. 2010;124(9):1010-3. Available from: https://www.cambridge.org/core/article/oralvoriconazole-for-invasive-fungal-skull-baseinfection/23E1316F77864E94F17B9CB6C1C6DBFD. Date accessed: 5 August 2019

14. Akhaddar A, Gazzaz M, Albouzidi A, Lmimouni B, Elmostarchid B, Boucetta M. Invasive Aspergillus terreus sinusitis with orbitocranial extension: case report. Surg Neurol [Internet]. 2008;69(5):490-5. Available from:

http://www.sciencedirect.com/science/article/pii/S0090 301907003977. Date accessed: 5 August 2019.

15. Levin LA, Avery R, Shore JW, Woog JJ, Sullivan Baker A. The spectrum of orbital aspergillosis: a clinicopathological review. Surv Ophthalmol [Internet]. 1996;41(2):142-54. Available from: http://www.sciencedirect.com/science/article/pii/S0039 62579680004X. Date accessed: 5 August 2019 